\title{
Nonlinear Transport in Mesoscopic Systems
}

\author{
T. K. $\mathrm{Ng}^{(\mathrm{a})}$ \\ AT\& T Bell Laboratories, Murray Hill, New Jersey 07974 \\ (Received 10 April 1991)
}

\begin{abstract}
A simple ansatz is proposed for the statistical mechanics governing nonlinear steady-state transport in mesoscopic systems. It is shown that unexpected, novel phenomena arise as a natural prediction of the ansatz. In particular, we discuss the situation of resonant tunneling where the nonlinear effect is expected to be strongest.
\end{abstract}

PACS numbers: $72.15 . \mathrm{Gd}, 72.10 . \mathrm{Bg}, 73.50 . \mathrm{Bk}$

In the past few years, there has been tremendous progress in the understanding of transport in systems with dimensions small compared to an equilibration length (mesoscopic systems), from both the theoretical [1-6] and the experimental sides [7-11]. In the linear-response regime, much of the novel phenomena associated with these systems can be understood in terms of elastic scattering of incident electrons from one probe into another, and the conductance of these systems can be expressed solely in terms of the transmission and reflection coefficients of electrons on the Fermi surface $[1,4,6]$.

However, experimentally it is often found that the transmission and reflection coefficients $T$ and $R$ can vary strongly (e.g., in resonant tunneling experiment) on a scale of less than a few meV and it is easy to reach the nonlinear regime where voltage differences between different probes are large compared with the energy range where $T$ varies substantially $[12,13]$. In this case a theory which incorporates the large current flow and/or voltage difference in the system at the starting point is needed to describe the experimental situation correctly.

Unfortunately there exists at present no general theory of statistical mechanics for systems far from equilibrium. The existing approaches on nonlinear transport in mesoscopic systems are all based on the perturbation expansion taking electric field as a small parameter [14]. The usefulness of this approach is not certain for systems far from equilibrium. Furthermore, in this approach current is always calculated as a response of the system to an applied external potential, whereas in real experiments it often happens that currents are driven through samples and the resulting potential differences between leads are measured. It is not clear whether these two approaches are identical in the nonlinear regime where the $I-V$ relation may not be unique.

The answer to these questions depends on the correct statistical-mechanics rules governing nonequilibrium mesoscopic systems and is unknown at present. In this Letter, we shall study this problem by proposing an ansatz for the statistical mechanics governing a mesoscopic system away from equilibrium. We shall consider the situation where voltage differences between different probes are large compared with the energy range where $T$ 's and $R$ 's vary, but small compared with the characteristic energy of the reservoirs themselves, so that the reservoir's behavior can be described by the usual linear- response theory. For simplicity we shall also restrict ourselves to noninteracting electrons in the present paper.

The ansatz we propose is that under the above conditions, a mesoscopic system in a nonequilibrium but steady-state situation can be treated as a system in equilibrium, except that the boundary conditions are modified. In the present case, the modified boundary conditions are constraints on the amount of current flowing through the probes, or voltage differences between different probes.

To put the ansatz in more concrete terms, we consider a mesoscopic system connected by $J$ probes to $J$ reservoirs and with $M_{j}$ channels at each probe $j$. The presence of an integer number of channels per probe is due to the quantization of electronic motions transverse to the probes $[2,4]$. We shall characterize each channel $n$ in probe $j$ by a dispersion [4] $\varepsilon_{j n}(k)=\varepsilon_{j n}(0)+(\hbar k)^{2} / 2 m^{*}$, where $\hbar k$ is the momentum associated with electronic motions parallel to the probe. The Hamiltonian characterizing the system is thus

$$
H_{0}=\sum_{k, i, n} \varepsilon_{i n}(k) \hat{C}_{i n}^{\dagger}(k) \hat{C}_{i n}(k) \text {, }
$$

where $\hat{C}_{i n}^{\dagger}(k)$ creates an exact scattering state $\psi_{\text {in }}(k)$ of the Hamiltonian incoming from channel $n$ in probe $i$ and with momentum $\hbar k$. Notice that spin is being neglected in our present discussion but can be included in a straightforward generalization of the present theory.

The eigenstates $\psi_{i n}(k)$ 's can be related to the incoming plane-wave states $\phi_{i n}(k)=e^{i\left(k x_{i n}+\delta_{i n}\right)}$ through the $S$ matrix,

$$
\begin{aligned}
\psi_{i n}(k) & =\hat{S} \phi_{i n}(k) \\
& =\sum_{j, m}\left[\delta_{i j} \delta_{n m} e^{i k x_{i n}}+\tilde{S}_{i j, m m}(k) e^{-i k x_{j m}}\right] e^{i \delta_{i n}},
\end{aligned}
$$

where $\tilde{S}_{i j, n m}(k)$ is an $S$-matrix element describing scattering of incoming wave $\phi_{\text {in }}(k)$ to outgoing wave in channel $m$ in probe $j$. Notice that we have introduced a phase $\delta_{i n}$ in $\psi_{i n}(k)$ to keep track of the phase coherence of the system. The phase $\delta_{i n}$ is a result of random thermal fluctuations at reservoir $i$ and is completely uncorrelated between different reservoirs and different channels. However, once the electron leaves the reservoir, the phase is fixed since there are no other phase breaking mechanisms in the sample itself [1-6].

The dc current operator in probe $i$ is 


$$
\hat{j}_{i}=\frac{e}{L} \sum_{n} \int_{0}^{L} \hat{j}\left(x_{i n}\right) d x_{i n}
$$

where $L$ is the length of the probe $(L \rightarrow \infty)$, and $\hat{j}\left(x_{i n}\right)=(i \hbar / 2 m)\left[\hat{\psi}^{\dagger}\left(x_{i n}\right) \partial \hat{\psi}\left(x_{i n}\right) / \partial x_{i n}-\hat{\psi}\left(x_{i n}\right) \partial \hat{\psi}^{\dagger}\left(x_{i n}\right) / \partial x_{i n}\right]$ is the current operator at position $x$ in probe $i$, channel $n$. Expressing $\hat{\psi}\left(x_{i n}\right)$ in terms of eigenstates $\psi_{i n}(k)$ and using Eq. (2), we find after some algebra

$$
\begin{aligned}
\hat{j}_{i}= & e \hbar \sum_{\substack{k, j, j^{\prime} \\
m, m^{\prime}, n}} \frac{k}{m^{*}}\left[\delta_{j i} \delta_{j^{\prime} i} \delta_{m n} \delta_{m^{\prime} n}-\tilde{S}_{j^{\prime} i, m^{\prime} n}(k) \tilde{S}_{i j, n m}^{*}(k)\right] e^{i\left(\delta_{j^{\prime} m^{\prime}}-\delta_{j m}\right)} \hat{C}_{j m}^{\dagger}(k) \hat{C}_{j^{\prime} m^{\prime}}(k) \\
= & e \hbar \sum_{\substack{k, m, n \\
m^{*}}} \frac{k}{m^{*}}\left[\delta_{n m}-R_{i i, n m}(k)\right] \hat{C}_{i m}^{\dagger}(k) \hat{C}_{i m}(k)-e \hbar \sum_{\substack{m, j \neq i \\
m, n}} \frac{k}{m^{*}} T_{i j, n m}(k) \hat{C}_{j m}^{\dagger}(k) \hat{C}_{j m}(k) \\
& -e \hbar \sum_{\substack{k, j \neq j^{\prime} \\
m, m^{\prime}, n}} \frac{k}{m^{*}} \tilde{S}_{j^{\prime} i, m^{\prime} n}(k) \tilde{S}_{i j, n m}^{*}(k) e^{i\left(\delta_{j^{\prime} m^{\prime}}-\delta_{j m}\right)} \hat{C}_{j m}^{\dagger}(k) \hat{C}_{j^{\prime} m^{\prime}}(k),
\end{aligned}
$$

where $R_{i i, n m}(k)=\left|\tilde{S}_{i i, n m}(k)\right|^{2}$ and $T_{i j, n m}=\left|\tilde{S}_{i j, n m}\right|^{2}$ are the reflection and transmission coefficients defined as usual $[1,2,4]$. The phase incoherence condition between reservoirs $\left\langle e^{i\left(\delta_{j^{\prime} m^{\prime}}-\delta_{j m}\right)}\right\rangle=\delta_{j j^{\prime}} \delta_{m m^{\prime}}$ implies that the last term in Eq. (4) can be neglected when computing the current expectation value $j_{i}=\left\langle\hat{j}_{i}\right\rangle$. However, it may have a finite contribution when computing expectation values of objects involving more than one current operator, as noted by Büttiker in computing shot noises [15] in mesoscopic systems.

The constraints in currents and/or voltage differences are imposed by adding Lagrange multiplier terms to the Hamiltonian. In an experiment where currents passing through the sample are fixed, the effective Hamiltonian becomes

$$
H_{\mathrm{eff}}=H_{0}+\sum_{i} \lambda_{i}\left(\hat{j}_{i}-J_{i}\right)
$$

where $J_{i}$ 's are the imposed current values in probe $i$. Substituting Eq. (4) into Eq. (5) and after some simple algebra, we obtain

$$
H_{\mathrm{eff}}=\sum_{k, i, n} E_{i n}(\lambda, k) \hat{C}_{i n}^{\dagger}(k) \hat{C}_{i n}(k)-\sum_{i} \lambda_{i} J_{i},
$$

where

$$
\begin{aligned}
& E_{i n}(\lambda, k)=\varepsilon_{i n}(k)+\frac{\hbar k}{m^{*}}\left(\lambda_{i}\left[1-R_{i n}(k)\right]\right. \\
& \left.-\sum_{j \neq i} \lambda_{j} T_{j i, n}(k)\right), \\
& R_{i n}(k)=\sum_{m} R_{i i, n m}(k),
\end{aligned}
$$

and

$$
T_{j i, n}(k)=\sum_{m} T_{j i, n m}(k) .
$$

Thus our ansatz simply says that the properties of the system under the current constraint (5) can be treated as a system in thermal equilibrium described by the effective Hamiltonian (6), with the Lagrange multipliers $\lambda_{i}$ 's determined by the physical constraint $\left\langle\hat{j}_{i}\right\rangle=J_{i}$.

The chemical potentials (voltages) $\mu_{i}$ 's at different reservoirs can be related to the corresponding particle densities $n_{i}$ through the usual relation [4],

$$
\Delta n_{i}=N_{F} \Delta \mu_{i}
$$

where $\Delta \mu_{i}$ and $\Delta n_{i}$ are the derivations from the corresponding equilibrium values $n_{0}$ and $\mu_{0}$ for $J_{i} \equiv 0$, and $N_{F}$ is the density of states of the reservoirs. This simple relation is valid because of our assumption that $\Delta \mu$ 's are small compared with the characteristic energies of the reservoirs. The particle densities at different reservoirs are given by the usual expression [4] $n_{i}$ $=\sum_{k, n}\left\langle\hat{C}_{i n}^{\dagger}(k) C_{i n}(k)\right\rangle$, except that the expectation value is now taken with respect to the effective Hamiltonian (6). Thus for a given set of values $J_{i}$ 's, we first solve the constraint equation $\left\langle\hat{j}_{i}\right\rangle=J_{i}$ to determine the Lagrange multipliers $\lambda_{i}$ 's, then use the effective Hamiltonian (6) to compute the densities $n_{i}$ 's, and then use Eq. (7) to determine the voltage differences $\Delta_{\mu_{i}}$ 's between different probes.

We can also imagine another possible experimental situation where the chemical potentials at different reservoirs $\mu_{i}$ 's are first fixed and the resulting currents are measured. The effective Hamiltonian now takes the form

$$
H_{\mathrm{eff}}=H_{0}+\sum_{i} \mu_{i}^{\prime}\left(\hat{n}_{i}-\rho_{i}\right)
$$

where $\rho_{i}$ is the particle density at reservoir $i$ which is related to the chemical potential through Eq. (7). Notice that the effective Hamiltonian (8) is very different from the corresponding effective Hamiltonian (6), implying that the $I-V$ characteristics measured in this situation may be different from what is found in the first experimental situation, even though the underlying Hamiltonian of the system remains the same.

In the linear-response regime, one can replace $R_{\text {in }}(k)$ and $T_{j i, n}(k)$ by the corresponding values on the Fermi surface $[1,4]$ and it is easy to show that both Eqs. (6) and (8) reduce to Büttiker's result, $J_{i}=(e / h)\left[\left(M_{i}\right.\right.$ $\left.\left.-R_{i i}\right) \Delta \mu_{i}-\sum_{j \neq i} T_{i j} \Delta \mu_{j}\right]$, where $R_{i i}=\sum_{n} R_{i n}\left(k_{F}\right), T_{i j}$ $=\sum_{n} T_{i j, n}\left(k_{F}\right)$, and $M_{i}$ is the total number of channels in probe $i$.

To understand the physics in the nonlinear regime, we study a two-probe (left and right) system with only one scattering channel at each probe at zero temperature. We shall first consider the situation where the potential 
difference between the two probes is fixed and the current flowing through is measured. In this case, we have

$$
\begin{aligned}
H_{\mathrm{eIf}}= & \sum_{k}\left[\varepsilon(k)-\mu_{l}\right] \hat{C}_{l}^{\dagger}(k) \hat{C}_{L}(k) \\
& +\sum_{k}\left[\varepsilon(k)-\mu_{R}\right] \hat{C}_{R}^{\dagger}(k) \hat{C}_{R}(k),
\end{aligned}
$$

and the "ground" state of the system consists of left and right Fermi seas filled up to chemical potentials $\mu_{L}$ and $\mu_{R}$, respectively. The current $I$ flowing through the system is

$$
\begin{aligned}
I= & e \hbar \sum_{k}\left(k / m^{*}\right) T(k)\left[n_{L}(k)-n_{R}(k)\right] \\
& \sim(e / h) \int_{\mu_{R}}^{\mu_{L}} d \varepsilon T(\varepsilon),
\end{aligned}
$$

where we have assumed $\mu_{L}>\mu_{R}$ and approximate $k \sim k_{F}$ in the last expression. In particular, we find $d I / d V \sim T$ gives the transmittance of the electrons from one probe into the other, as is expected from "naive" consideration of nonlinear transport [16].

The situation is, however, very different in the situation when the current is fixed first. In this case, we have

$$
\begin{aligned}
H_{\mathrm{eff}}= & \sum_{k}[\varepsilon(k)-\lambda T(k)-\mu] \hat{C}_{l}^{\dagger}(k) \hat{C}_{L}(k) \\
& +\sum_{k}[\varepsilon(k)+\lambda T(k)-\mu] \hat{C}_{R}^{\dagger}(k) \hat{C}_{R}(k),
\end{aligned}
$$

where $\mu$ is the average chemical potential which fixes the total number of electrons in the system and $\lambda$ is the Lagrange multiplier determined by the current constraint $\langle\hat{j}\rangle=I$. To proceed further we assume that $T$ has the resonant tunneling form

$$
T(k) \sim \frac{a \Delta}{\left[\varepsilon(k)-\varepsilon_{0}\right]^{2}+\Delta^{2}},
$$

where $a \leq 1$, and $\varepsilon_{0}$ and $\Delta$ are the position and width of the resonance, respectively. Notice that the effective dispersion $\varepsilon(k)-\lambda T(k)$ develops a minimum around $\varepsilon(k) \sim \varepsilon_{0}$ when $\lambda a$ is large enough, because of the maximum in $T(k)$ at $\varepsilon(k)=\varepsilon_{0}$. Thus for some values of $\lambda$ (or $I)$ and $\mu$, one may have the seemingly very awkward situation where the left-coming electronic states $k$ with $\varepsilon(k) \sim \varepsilon_{0}$ will be filled up first, whereas some states with lower "true" energy $\varepsilon(k)$ remain unoccupied, leaving a "gaplike" structure in the occupation number of the leftcoming states, in contrast to the previous situation where states are filled up in order of their true energy.

The physical origin of this strange behavior can be understood in terms of our ansatz which reduces the nonlinear transport problem to the problem of minimization of the total energy of the system with a fixed current at zero temperature. Although states at $\varepsilon(k) \sim \varepsilon_{0}$ have higher energy than those states with smaller $k$ 's, they also allow a larger current flow. Thus for a fixed amount of current, it may be energetically more favorable to fill up these "resonant" states first, in exchange for smaller chemical potential differences between the reservoirs.
It is obvious that the $I-V$ characteristic of the system obtained in this way will in general be different from the one obtained in the previous situation where the chemical potentials are fixed first because of the very different way of distributing electrons. This "nonunique $I-V$ " behavior can be understood more generally by considering the total free energy $F(I, \Delta \mu)$ of the system as function of given current $I$ and potential difference $\Delta \mu$ between the two leads. The absolute minimum of $F(I, \Delta \mu)$ occurs at $I=\Delta \mu=0$, i.e., equilibrium. In experiment where $\Delta \mu$ is fixed, $I$ is determined by the minimum in $F(I, \Delta \mu)$ with fixed $\Delta \mu$, and vice versa. The difference in the $I-V$ characteristic for the two experiments is a consequence of the fact that the values $(I, \Delta \mu)_{\Delta \mu}$ where $F(I, \Delta \mu)$ is minimum for a fixed $\Delta \mu$ do not coincide in general with the values $(I, \Delta \mu)$, where $F(I, \Delta \mu)$ is a minimum for fixed I. The two minima coincide exactly only in the linearresponse regime. Notice that the minima in $F(I, \Delta \mu)$ for a fixed nonzero value of $I$ or $\Delta \mu$ are not true minima for the free energy of the system but are saddle points in the $(I, \Delta \mu)$ energy diagram which are minima only along the "constrained" lines with fixed value of $I$ or $\Delta \mu$ characterizing the nonequilibrium system. With more probes connected to the sample the consideration is similar.

In comparing theory with experiment, it must be kept in mind that it is not true that simply driving currents through the sample would automatically match the "fixed current" condition described by Eq. (6). For example, if the electrons were allowed to relax to local equilibrium before reaching the sample, the momentum distribution would be independent of the scattering properties of the sample and would probably follow the distribution described by Eq. (8). It is not clear at present what are the correct experimental conditions corresponding to the fixed current or fixed voltage situations. Another difficulty is that in reality the scattering potential which determines the transmittance and reflectance of the system depends also on the voltage differences and currents in the system $[6,14]$. For a noninteracting system, voltage differences between different probes introduce additional electric fields in the sample modifying the scattering potential $[6,14]$. However, if the probes are near-perfect conductors, the electric fields are essentially constrained to the sample region and can be incorporated into our formulation by considering electric-field-dependent scattering matrices. The dependence of $T$ 's and $R$ 's on the potential differences between probes further complicates the analysis of the $I-V$ curves. However, the main prediction of our theory, the nonuniqueness of the $I-V$ characteristic, still remains unaltered since this phenomenon originates from the different ways of minimizing the free energy under different experimental conditions and is independent of the microscopic details of the scattering matrix.

Theoretically, it is expected that this nonuniqueness effect will be strongest in systems with strong fluctuations in transmittance over a narrow energy range. To test this effect we consider the two-probe, single-channel system 


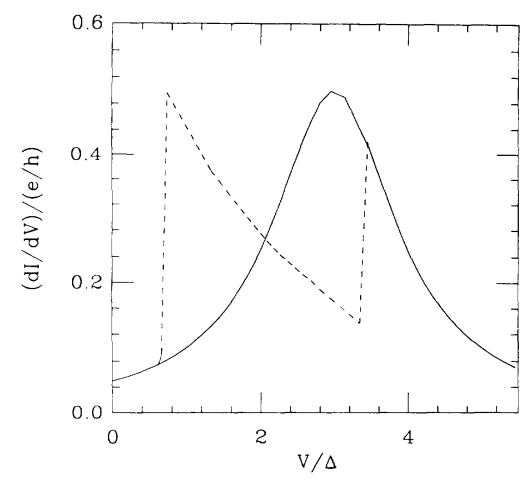

FIG. 1. $d I / d V$ curves for a two-probe, resonant-tunneling system at zero temperature for fixed $\mu_{R}=\varepsilon_{0}-3 \Delta$. The solid line gives the result when the voltage difference between the two probes is first fixed and the current $I$ measured. The dashed line gives the opposite situation.

with transmittance given by Eq. (11) (with $a=0.5$ ) and compute the $I-V$ curve numerically at zero temperature for both the situation with fixed currents and that with fixed voltage differences (assuming that the dependence of $T$ on $\Delta \mu$ is negligible). The resulting $d I / d V$ curve as a function of $V=\mu_{L}-\mu_{R}$ for fixed $\mu_{R}=\varepsilon_{0}-3 \Delta$ is shown in Fig. 1 for both situations (the solid line is for fixed voltage difference, the dashed line is for fixed current). The drastic difference between the two situations is apparent. The "double peak" structure in $d I / d V$ for the fixed current situation is associated with the creation of the gap in the occupation number of left-coming states and the closing of the gap region when voltage difference is large enough. Notice that the two curves coincide for small values of $\Delta \mu$ (linear-response regime) and that the area under the two curves is the same, reflecting the fact that the same set of electron states is involved in the two situations. In a realistic situation more than one channel exists but qualitative behavior similar to Fig. 1 is still expected if resonant behavior similar to Eq. (11) is found for the total transmittance $T_{n}=\sum_{m} T_{n m}$ for some channel $n$ into the opposite probe, and if the background conductance is small enough.

We now make a few comments on the physical meaning of the ansatz proposed in this paper. The basic assumption behind the ansatz is that the statistical properties of mesoscopic systems in a nonequilibrium steady state are the same as in an equilibrium state. Notice that nonlinearity in mesoscopic transport is a direct consequence of the quantum interference effect, and thermorelaxation of the system occurs only at the probes joining the sample to the reservoirs, i.e., the relaxation processes are completely nonlocal. This is in deep contrast with nonlinear transport in macroscopic systems where the system is out of the thermoequilibrium and dissipation within the sample is crucial in maintaining the nonequi- librium steady state $[17,18]$. Our thermodynamic ansatz which describes the current-carrying sample as a dissipationless equilibrium state with minimum free energy (like a superconductor carrying supercurrent) may be valid in mesoscopic systems where quantum coherence is maintained throughout the sample, but is certainly not valid in describing nonlinear transport in macroscopic systems where dissipation is crucial. The main discovery of the present paper is that a simple ansatz like the one we propose here can produce rich and unexpected features in the behavior of nonequilibrium mesoscopic systems. The validity of our prediction depends on how reservoirs work in reality which is largely unknown at present. Thus experimental tests of the theory would provide us with information on how reservoirs work in mesoscopic systems.

The author thanks H. Baranger, P. de Vegvar, and P. A. Lee for helpful discussions.

(a) Present address: Department of Physics, Hong Kong University of Science and Technology, Clear Water Bay Road, Kowloon, Hong Kong.

[1] R. Landauer, Philos. Mag. 21, 863 (1970).

[2] See, for example, Y. Imry, in Directions in Condensed Matter Physics, edited by G. Grinstein and E. Mazenko (World Scientific, Singapore, 1986).

[3] P. A. Lee and A. D. Stone, Phys. Rev. Lett. 55, 1622 (1985).

[4] M. Büttiker, Phys. Rev. Lett. 57, 1761 (1986).

[5] R. A. Serota, M. Ma, and B. Goodman, Phys. Rev. B 37, 6540 (1988).

[6] H. U. Baranger and A. D. Stone, Phys. Rev. B 40, 8169 (1989).

[7] C. P. Umbach, S. Washburn, R. A. Laibowitz, and R. A. Webb, Phys. Rev. B 30, 4048 (1984).

[8] R. A. Webb, S. Washburn, C. Umbach, and R. A. Laibowitz, Phys. Rev. Lett. 54, 2996 (1985).

[9] S. B. Kaplan and A. Harstein, Phys. Rev. Lett. 56, 2403 (1986).

[10] A. Benoit, C. P. Umbach, R. B. Laibowitz, and R. A. Webb, Phys. Rev. Lett. 58, 2343 (1987).

[11] S. Washburn, A. B. Fowler, H. Schmid, and D. Kern, Phys. Rev. Lett. 61, 2801 (1988).

[12] R. A. Webb, S. Washburn, and C. P. Umbach, Phys. Rev. B 37, 8455 (1988).

[13] P. G. N. deVegvar, G. Timp, P. M. Mankiewich, J. E. Cunningham, R. Behringer, and R. E. Howard, Phys. Rev. B 38, 4326 (1988).

[14] A. I. Larkin and D. E. Khmelnitskii, Zh. Eksp. Teor. Fiz. 91, 1815 (1986) [Sov. Phys. JETP 64, 1075 (1986)].

[15] M. Büttiker, Phys. Rev. Lett. 65, 2901 (1990).

[16] S. J. Bending and M. R. Beasley, Phys. Rev. B 35, 2548 (1987).

[17] K. K. Thornber and R. P. Feynman, Phys. Rev. B 1, 4099 (1970)

[18] X. L. Lei and C. S. Ting, Phys. Rev. B 32, 1112 (1985). 